\title{
Raman Amplification in Plasma: Thermal Effects
}

\author{
John Farmer, Bernhard Ersfeld and Dino Jaroszynski \\ Department of Physics, SUPA, University of Strathclyde, \\ 107 Rottenrow East, Glasgow, G4 ONG, UK
}

\begin{abstract}
The impact of thermal effects on Raman amplification in plasma is investigated theoretically. It is shown that damping and the shift in plasma resonance at finite temperature can alter the evolution of an amplified pulse. It is shown that pulse compression can occur which is not predicted by the cold plasma model. Although thermal effects can lead to a reduction in the efficiency of the interaction, this can be avoided by using a chirped pump. In this case these effects can be beneficial, suppressing the development of a train of pulses behind the amplified seed, as observed in the cold plasma model.
\end{abstract}

Keywords: Raman, laser, amplification, plasma, thermal, Bohm-Gross, heating, damping, Langmuir

PACS: $52.38 . \mathrm{Bv}$

\section{INTRODUCTION}

Raman amplification in plasma is a potential method for the creation of intense, ultrashort laser pulses [1]. Two laser pulses of different frequencies are coupled by the motion of the plasma electrons, allowing energy transfer between the two. A counterpropagating geometry allows a short seed pulse to be amplified by a long pump beam, allowing the seed to grow to intensities greater than that of the pump [2].

Raman amplification is an attractive prospect as it could potentially lead to significant reductions in both the size and cost of high power laser systems. Unlike conventional optical media, plasma has no damage threshold, thus removing the need for chirped pulse amplification and so avoiding the need for compression gratings, which become large and expensive at high powers. Further, plasma has the potential of offering high efficiencies over shorter distances than conventional optical amplifiers.

Although such sources will have uses across a wide range of science and technology applications, it is of particular interest to the laser-plasma accelerator community, who already have much of the skills and equipment necessary to exploit this technique.

\section{THEORY}

When two counterpropagating laser pulses of different frequencies collide in plasma, the resulting beat wave will act upon the plasma electrons through the ponderomotive force, driving electrons toward areas of lower intensity. If the lasers are detuned by the plasma frequency a plasma wave will be excited. The resulting density perturbation will act as a moving Bragg grating which scatters photons from the higher frequency laser pulse 
to the lower, amplifying the latter. In this way, a short, low intensity seed pulse can be amplified using a long counterpropagating pump beam.

In the linear regime [1] the amplification bandwidth is very narrow, due to the harmonic motion of the plasma electrons. Pump depletion is negligible and seed growth exponential. However, the density perturbation that is created continues to scatter the pump after the probe has passed, leading to an increase in the duration of the amplified seed proportional to the propagation distance.

In the pump-depletion regime [3] depletion of the pump is sufficiently high as to suppress amplification towards the rear of the seed, leading to pulse compression and spectral broadening. The amplified seed develops as a train of short pulses of decreasing intensity, due to Burnham-Chiao ringing [5], which occurs when energy is transferred from the probe back in to the pump.

It has been shown [4] that using a chirped pump allows a pulse to be amplified in the linear regime without the increase in duration observed when using a monochromatic pump. The amplification bandwidth is increased due to the varying frequency of the pump. The different frequency components will be in phase close to the head of the seed, but further behind they will destructively interfere, preventing pulse lengthening. The peak intensity grows linear with distance: while each frequency component is amplified exponentially, they will only interact for a finite time before falling out of resonance. The same effect can be achieved using a plasma density gradient.

\section{Impact of thermal effects}

Damping and the plasma response at finite temperature have several implications for Raman amplification in plasma.

At finite temperature, contributions from the electron pressure lead to an increase in the resonant frequency of the plasma. This Bohm-Gross shift will alter the resonance conditions of the Raman process.

In addition, the plasma wave will be subject to Landau damping. Electrons with thermal velocities close to the phase velocity of the plasma wave will exchange energy with the wave. Those with velocities just below the phase velocity of the plasma wave will be accelerated, while those just above will be decelerated. Analogues can be drawn with plasma wakefield acceleration. For a Maxwellian distribution, the net effect is for the plasma wave to lose energy.

Collisional damping of the laser pulses will not have a significant direct impact on their intensities for the parameters used in current experiments (e.g. [2]). However, it will lead to an increase in the plasma temperature. The plasma wave, on the other hand, may be significantly damped, due to a combination of collisional damping and Landau damping, which will also contribute to an increase in temperature. 


\section{SIMULATION MODEL}

Simulations have been carried out using a 1-dimensional fluid model, based on the slowly varying envelope approximation for Raman amplification [1], modified to take into account thermal effects.

The resonant frequency of the plasma at finite temperatures is given by

$$
\omega_{p}^{\prime}=\sqrt{\omega_{p}^{2}+3 \theta c^{2} k_{\|}^{2}}
$$

where $\omega_{p}=\sqrt{n_{e} e^{2} / \varepsilon_{0} m}$ is the plasma frequency, corresponding to the cold plasma resonance, $k_{\|}$the wavenumber of the Langmuir wave and $\theta=\frac{3 k_{B} T}{m c^{2}}$ the normalised temperature. $e$ and $m$ are, respectively, the electron charge and mass, $\varepsilon_{0}$ the permittivity of free space, $c$ the vacuum speed of light, $k_{B}$ the Boltzmann constant and $T$ the temperature.

The plasma will be heated according to

$$
\frac{\partial \theta}{\partial t}=v_{\perp}\left(\left|a_{0}\right|^{2}+\left|a_{1}\right|^{2}\right) / 3+\left(v_{\|}+v_{L}\right)|f|^{2} / 3
$$

where $a_{0,1}=\frac{E_{0,1} e}{m \omega_{0,1} c}$ are the reduced vector potentials of the pump and probe, respectively, $E_{0,1}$ and $\omega_{0,1}$ are the associated transverse electric field and frequency. $f=\frac{E_{z} e}{m \omega_{p} c}$ is a normalised form of the longitudinal electric field $E_{z} \cdot v_{\perp, \|}$ are the transverse and longitudinal collisional damping rates, and $v_{L}$ the Landau damping rate.

The transverse and longitudinal collisional damping rates are given by substituting the laser and plasma frequencies, respectively, into the expression

$$
v=\frac{Z e^{2} \omega_{p}^{2}}{6(2 \pi)^{3} / 2 \varepsilon_{0} m c^{3}}\left[\ln \left(\frac{4 \theta m c^{2}}{\hbar \omega}\right)-\gamma\right]
$$

where $\hbar$ is Planck's constant divided by $2 \pi$ and $\gamma$ is Euler's constant. The Landau damping rate is approximated by

$$
v_{L} \approx \sqrt{\frac{\pi}{8}} \frac{\omega_{p}^{4}}{k_{\|}^{3} c^{3}} \theta^{-3 / 2} \exp \left(-\frac{\omega_{p}^{2}}{2 k_{\|}^{2} c^{2} \theta}-\frac{3}{2}\right)
$$

The modified envelope equations for the Raman process are then

$$
\begin{gathered}
\left(\frac{\partial}{\partial t}-c \frac{\partial}{\partial z}+\left(\frac{\omega_{0}}{\omega_{p}}\right)^{2} \frac{v_{\perp}}{2}\right) a_{0}=-\frac{\omega_{p} f^{*} a_{1}}{2} \\
\left(\frac{\partial}{\partial t}+c \frac{\partial}{\partial z}+\left(\frac{\omega_{0}}{\omega_{p}}\right)^{2} \frac{v_{\perp}}{2}\right) a_{1}=\frac{\omega_{p} f a_{0}}{2} \\
\left(\frac{\partial}{\partial t}+\frac{v_{\|}+v_{L}}{2}+i\left(\omega_{p}^{\prime}-\left(\omega_{0}-\omega_{1}\right)\right)\right) f=\frac{\omega_{0} a_{0}^{*} a_{1}}{2}
\end{gathered}
$$

These modified equations include damping of the plasma wave and the laser fields, while Eq. (7) also incorporates the shift in plasma resonance due to temperature. 


\section{RESULTS}

The simulation parameters were chosen to be similar to those used in recent experimental works [2]: Gaussian profile seed of FWHM $0.5 \mathrm{ps}$, peak intensity $1 \times 10^{12} \mathrm{~W} \mathrm{~cm}^{-2}$, $\omega_{1}=10 \omega_{p}$; flat top pump pulse, length $10 \mathrm{ps}$, intensity $1 \times 10^{14} \mathrm{~W} \mathrm{~cm}^{-2}, \omega_{0}=11 \omega_{p}$; plasma length $2 \mathrm{~mm}$, of density $n_{e}=1 \times 10^{19} \mathrm{~cm}^{-3}$ and initial temperature $5 \mathrm{eV}$.

Figs. 1.a,b show the simulation results for these parameters if thermal effects are not included. The pump depletion regime is accessed and the expected pulse compression and pi-pulse [3] profile are observed, with a peak intensity $\sim 2000$ times that of the input seed.

The inclusion of thermal effects, as shown in Figs. 1.c,d shows a reduction in the peak intensity, achieving an amplification of $\sim 500$ times. However, compression of the amplified pulse is still observed, and the profile is qualitatively similar to the pi-pulse. However, pump depletion reaches $\sim 30 \%$, and is seen to decrease after this point. This suggests that pump depletion is not the primary source of pulse compression.

This was verified by arbitrarily excluding pump depletion from the simulation model. Although this is clearly nonphysical, it serves as a useful way of examining the processes that contribute to the observed behaviour. Fig. 1.e shows the result of this modification: there is an increase in seed amplification, which is to be expected, but pulse compression still occurs. This behaviour cannot be accounted for using the cold plasma model. However, it can be explained simply by considering the Bohm-Gross frequency shift, as shown in Fig. 1.f. The plasma resonance varies with time, and so will have a similar effect to using a chirped pump or a plasma density gradient, allowing pulse compression.

It is important to note that thermal effects do not guarantee pulse compression. For example, at high initial temperatures, the shift in plasma resonance is smaller, allowing pulse lengthening as observed in the cold plasma model.

While the reduction in pulse length observed in Fig. 1.c is an interesting effect, the low efficiency probably makes this scenario unsuitable for practical applications. However, it is possible to compensate for the shift in plasma resonance through the use of a chirped pump pulse. Considering Eq. (7), it is clear that if $\omega_{0}$ increases with time to match the Bohm-Gross shift due to heating by the pump (as shown in Fig. 1.f), the two effects will cancel.

Fig. 2 shows the effect of using a pump with a linear chirpvarying from $11 \omega_{p}$ to $11.075 \omega_{p}$ over its 10ps duration. Although this is a rather rough match to the shift in resonance shown in Fig. 1.f, it can be seen that it is sufficient to significantly increase the efficiency of the interaction, giving an amplified pulse of peak intensity similar to that observed in the cold plasma model (Fig. 1.a).

While the peak efficiency is matched, the train of pulses behind the seed head is suppressed. This is due to imperfect matching of the shift in plasma resonance: although the shift due to heating by the unperturbed pump could be exactly matched, additional contributions due to damping of the plasma wave and seed cannot. This will lead to an increased shift in resonance behind the head of the seed, which will suppress BurnhamChiao ringing, both directly, through the shift in resonance, and indirectly, through the reduction in pump depletion. 
a

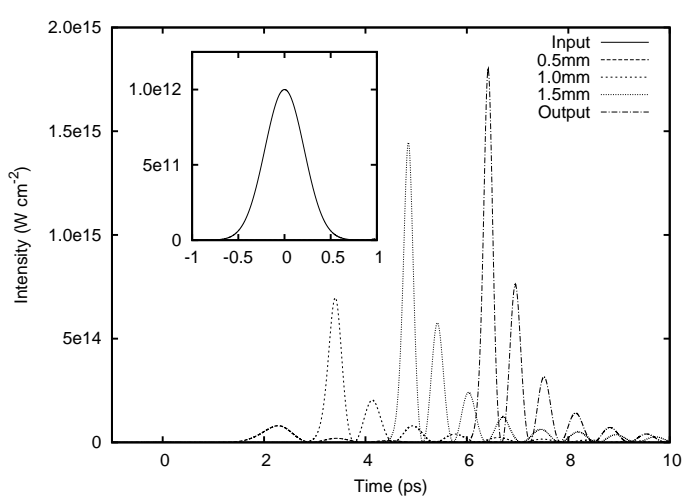

$\mathrm{c}$

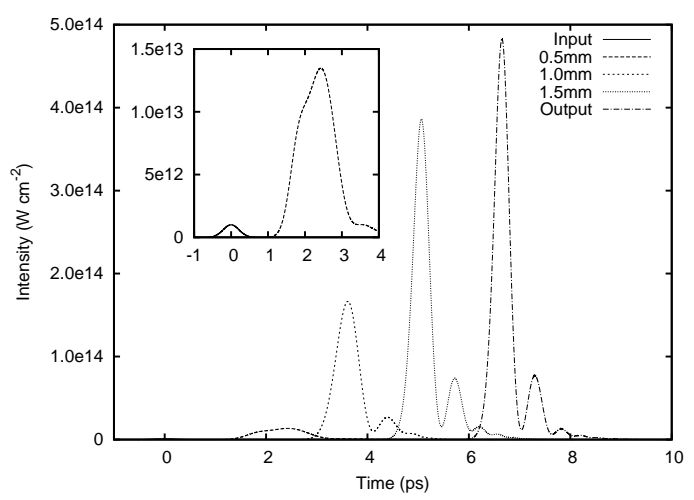

e

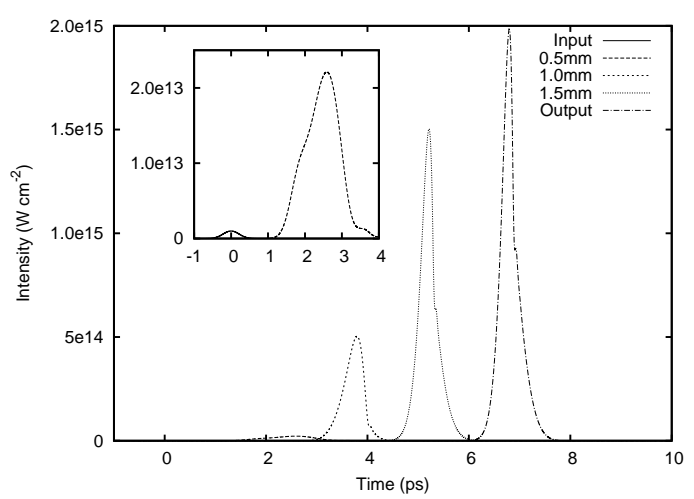

b

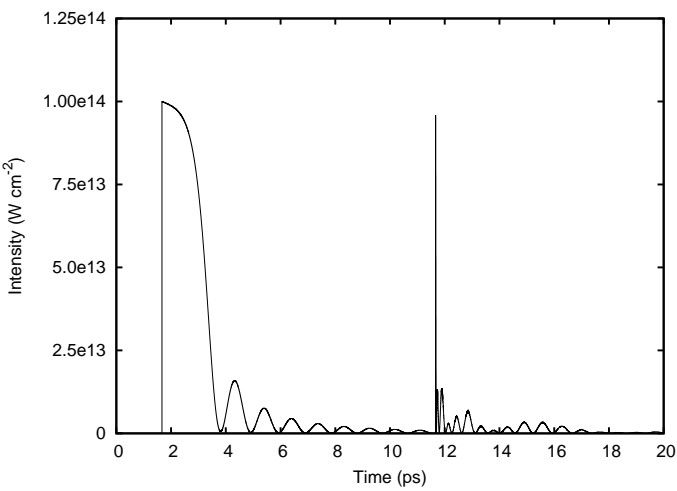

d

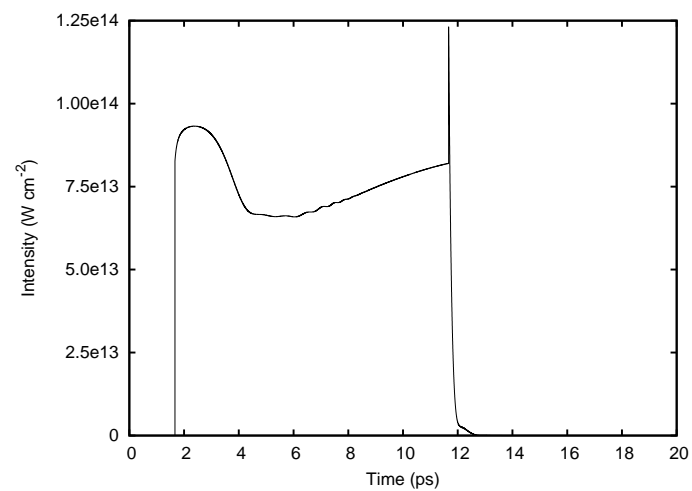

$\mathrm{f}$

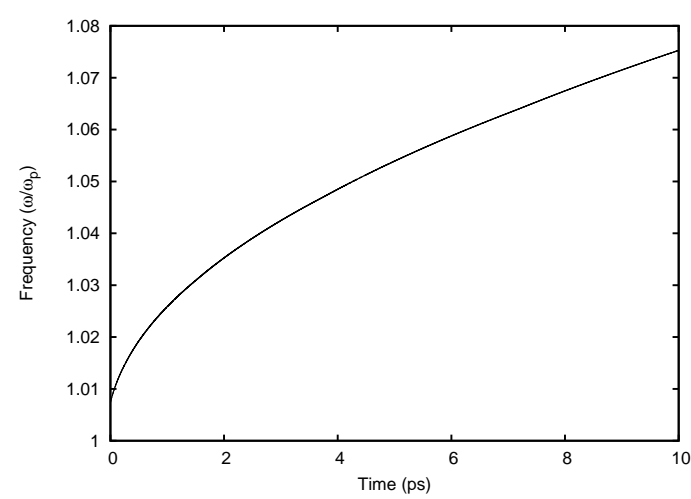

FIGURE 1. Simulation results illustrating the impact of thermal effects on Raman amplification.

(a) Seed evolution ${ }^{a}$ and (b) pump depletion ${ }^{b}$ using the cold plasma model.

(c) Seed evolution and (d) pump depletion using the thermal effects model.

(e) Seed evolution using the thermal effects model modified to remove the effects of pump depletion.

(f) Shift in plasma resonance due to heating of the plasma by the pump beam.

${ }^{a}$ The seed intensity observed at different positions in the plasma is plotted against time. For a given position, the head of the pulse will appear leftmost.

$b$ Temporal profile of the pump intensity as it leaves the plasma. 
a

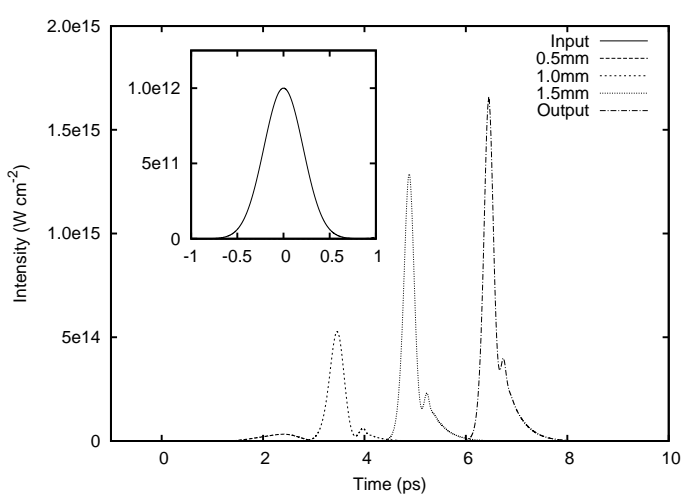

b

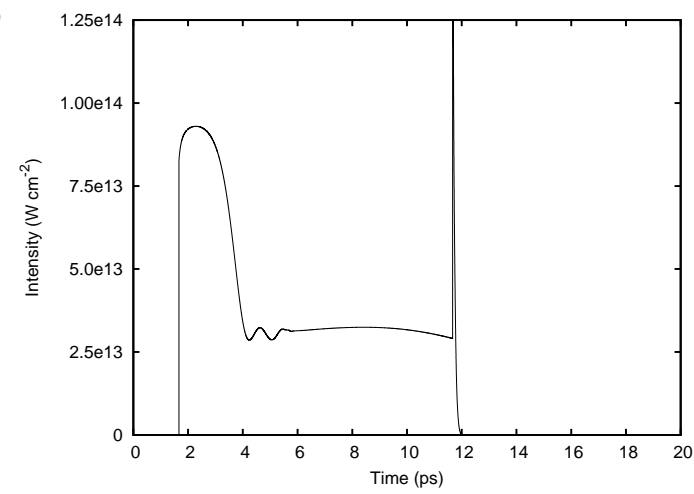

FIGURE 2. Effect of using a chirped pump to match the shift in resonance due to pump heating on (a) seed evolution and (b) pump depletion. Other parameters are as in Fig. 1.c.

\section{CONCLUSION}

The impact of thermal effects on Raman amplification has been investigated. The shift in plasma resonance over time due to heating by the pump acts as a chirp. For a monochromatic pump, this chirp leads to a reduction in the efficiency of the interaction and, in some cases, allows pulse compression without the need for pump depletion, a marked departure from the behaviour observed in the cold plasma model.

In order to avoid these effects, an up-chirp may be added to the pump beam to compensate for the shift in plasma resonance. The two chirps cancel, increasing the interaction efficiency. It is shown that this allows for an amplified pulse with peak intensity similar to that observed in the cold plasma model. However, additional heating due to damping of the plasma wave leads to a further shift in resonance behind the head of the seed. This limits pump depletion and suppresses Burnham-Chiao ringing, allowing a single intense pulse, rather than the series of pulses observed in the cold plasma model.

These results show that thermal effects can have a significant impact on Raman amplification, affecting not only the efficiency of the interaction, but altering the length and profile of the amplified pulse. For this reason thermal effects should be taken in to account both when planning experiments and analysing results.

\section{REFERENCES}

1. G. Shvets et al, Phys. Rev. Lett. 814879 (1998)

2. W. Cheng et al, Phys. Rev. Lett. 94045003 (2005)

J. Ren et al, Nat. Phys. 3732 (2007)

3. V. M. Malkin, G. Shvets and N. J. Fisch, Phys. Rev. Lett. 824448 (1999)

4. B. Ersfeld and D. A. Jaroszynski, Phys. Rev. Lett. 95165002 (2005)

5. D. C. Burnham and R. Y. Chiao, Phys. Rev. Lett. 188667 (1969) 